\title{
Tensor invariants for certain subgroups of the orthogonal group
}

\author{
Jan Draisma • Guus Regts
}

Received: 17 February 2012 / Accepted: 18 September 2012 / Published online: 9 November 2012

(C) Springer Science+Business Media New York 2012

\begin{abstract}
Let $V$ be an $n$-dimensional vector space, and let $O_{n}$ be the orthogonal group. Motivated by a question of B. Szegedy (J. Am. Math. Soc. 20(4), 2007), about the rank of edge connection matrices of partition functions of vertex models, we give a combinatorial parameterization of tensors in $V^{\otimes k}$ invariant under certain subgroups of the orthogonal group. This allows us to give an answer to this question for vertex models with values in an algebraically closed field of characteristic zero.
\end{abstract}

Keywords Edge connection matrix · Graph invariant · Partition function · Orthogonal group · Tensor invariants · Vertex model

\section{Introduction}

Let $\mathbb{F}$ be a field, and let $V$ be an $n$-dimensional vector space over $\mathbb{F}$ equipped with a nondegenerate symmetric bilinear form $\langle\cdot, \cdot\rangle$ equivalent to the standard form on $\mathbb{F}^{n}$. We use the superscript $*$ to indicate the duals of $\mathbb{F}$-vector spaces. Let $R=S V^{*}$ be the symmetric algebra generated by $V^{*}$, which we identify with the ring of polynomials on $V$. The orthogonal group $O_{n}$ is the group of invertible linear transformations of $V$ preserving the bilinear form. It has a natural action on $R$ and hence on $R^{*}$. Let $h \in R^{*}$ and define

$$
\operatorname{Stab}(h):=\left\{g \in O_{n} \mid g h=h\right\} .
$$

J. Draisma ( $\varangle)$

Eindhoven University of Technology and CWI, Amsterdam, The Netherlands

e-mail: j.draisma@tue.nl

G. Regts

CWI, Science Park, 1231098 XG Amsterdam, The Netherlands

e-mail: regts@cwi.nl 
The orthogonal group acts on $V^{\otimes k}$ for $k \in \mathbb{N}$. In this paper we give a combinatorial parameterization of the space of tensors that are invariant under $\operatorname{Stab}(h)$ for certain $h \in R^{*}$ when $\mathbb{F}$ is an algebraically closed field of characteristic zero. This interpretation can be seen as a generalization of the Brauer algebra, to which it reduces for $h=0$.

This work is motivated by a question of B. Szegedy concerning the rank of edge connection matrices of partition functions of vertex models. To state his question, we introduce some terminology. Let $\mathcal{G}$ be the (countable) set of isomorphism classes of finite, undirected graphs, allowing multiple edges, loops, and circles. Here a circle is a graph with one edge and no vertices. An $\mathbb{F}$-valued graph invariant is a map $f: \mathcal{G} \rightarrow \mathbb{F}$. We may think of $f$ as mapping graphs to $\mathbb{F}$ in such a way that isomorphic graphs are mapped to the same element. This allows us to use the term graph even when we mean an isomorphism class of graphs. In particular, we will somewhat inaccurately speak of the vertex set $V G$ and the edge set $E G$ of an element $G \in \mathcal{G}$.

Throughout this paper, we set $\mathbb{N}=\{0,1,2, \ldots\}$, and for $n \in \mathbb{N},[n]$ denotes the set $\{1, \ldots, n\}$.

Let $e_{1}, \ldots, e_{n} \in V$ be an orthonormal basis for $V$, and let $x_{1}, \ldots, x_{n} \in V^{*}$ be the associated dual basis. Then $R$ is just $\mathbb{F}\left[x_{1}, \ldots, x_{n}\right]$, the polynomial ring in $n$ variables. Following de la Harpe and Jones [4], we call any $h \in R^{*}$ an (F-valued) $n$-color vertex model. The vertex model can be considered as a statistical mechanics model, where edges serve as particles, vertices as interactions between particles, and colors as states or energy levels. The partition function of $h$ is the graph invariant $f_{h}: \mathcal{G} \rightarrow \mathbb{F}$ defined by

$$
f_{h}(G)=\sum_{\phi: E G \rightarrow[n]} \prod_{v \in V G} h\left(\prod_{e \in \delta(v)} x_{\phi(e)}\right)
$$

for any $G \in \mathcal{G}$. Here $\delta(v)$ is the multiset of edges incident with $v$. Several graph invariants are partition functions of vertex models. For example, the number of perfect matchings and also the number of homomorphisms into a fixed graph [12] (for complex-valued vertex models).

Remark 1 At first sight, this definition of $f_{h}$ seems to depend on the choice of orthonormal basis $e_{1}, \ldots, e_{n} \in V$. However, the following more conceptual interpretation shows that it really only depends on the bilinear form and on $h \in R^{*}=\left(S V^{*}\right)^{*}$ and that the definition can in principle be extended to symmetric bilinear forms for which (over algebraically nonclosed fields) no orthonormal basis exists. A graph $G=(V G, E G)$ gives rise to a polynomial function $\psi: V^{V G} \rightarrow \mathbb{F}$ by sending a tuple $\left(v_{i}\right)_{i \in V G}$ to $n$ to the power the number of circles in $G$ times the product of the expressions $\left\langle v_{i}, v_{j}\right\rangle$ over all noncircle edges $\{i, j\} \in E G$. Then $\psi$ is an element of the tensor power $R^{\otimes V G}$ (since coordinate rings of Cartesian products are tensor products of coordinate rings). Applying the element $h^{\otimes V G}$ to $\psi$ gives a number that is nothing but $f_{h}(G)$. By construction the function $\psi$ is invariant under the orthogonal group acting on $V^{V G}$, which implies that $f_{g h}=f_{h}$ for all $g \in O_{n}$. Many arguments 
Fig. 1 Gluing two $k$-fragments into a graph

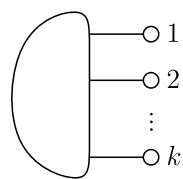

F

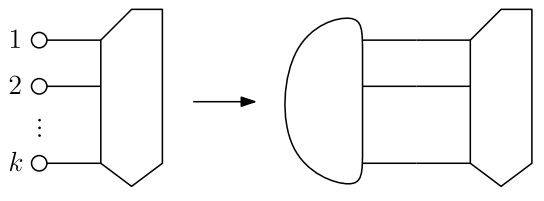

$H$

$F * H=H * F$

in this paper have coordinate-free analogues. For the sake of concreteness, however, we mostly work directly with formulas such as the one above.

We now introduce the concept of $k$-fragments for $k \in \mathbb{N}$. A $k$-fragment is a graph that has $k$ distinct degree-one vertices labeled 1 to $k$. These labeled vertices are called the open ends of the graph. The edge connected to an open end is called a half edge. Let $\mathcal{F}_{k}$ be the set of all $k$-fragments, so that $\mathcal{F}_{0}$ equals $\mathcal{G}$, the set of graphs without labels. Define a gluing operation $*: \mathcal{F}_{k} \times \mathcal{F}_{k} \rightarrow \mathcal{G}$ as follows: in the disjoint union of $F, H \in \mathcal{F}_{k}$, connect the half edges incident with open ends with identical labels to form single edges (with the labeled vertices erased); the resulting graph is denoted $F * H \in \mathcal{F}_{0}$; see Fig. 1 .

For any graph invariant $f$, let $M_{f, k}$ be the $\mathcal{F}_{k} \times \mathcal{F}_{k}$-matrix defined by

$$
M_{f, k}(F, H)=f(F * H)
$$

for $F, H \in \mathcal{F}_{k}$. This matrix is called the edge connection matrix of $f$. Edge connection matrices were used by Szegedy [12] to characterize graph invariants $f: \mathcal{G} \rightarrow \mathbb{R}$ that are partition functions of real-valued vertex models and by Schrijver [9] to characterize graph invariants $f: \mathcal{G} \rightarrow \mathbb{C}$ that are partition functions of complex-valued vertex models.

In [12] Szegedy asked for a characterization of the rank of $M_{f, k}$ for $k=1,2, \ldots$ if $f=f_{h}$ for some real-valued vertex model $h$. This question was answered by the second author in [8]. In this paper we give an answer to this question for $\mathbb{F}$-valued vertex models where $\mathbb{F}$ is an algebraically closed field of characteristic zero. Our characterization reads as follows.

Theorem 1 Let $\mathbb{F}$ be an algebraically closed field of characteristic zero, and let $h \in R^{*}$. Then there exists $h^{\prime} \in R^{*}$ such that $f_{h}(G)=f_{h^{\prime}}(G)$ for all $G \in \mathcal{G}$ and such that

$$
\operatorname{rk}\left(M_{f_{h}, k}\right)=\operatorname{dim}\left(V^{\otimes k}\right)^{\operatorname{Stab}\left(h^{\prime}\right)} .
$$

The organization of this paper is as follows. In Sect. 2 we compare the real case with the algebraically closed case. Section 3 contains some framework and preliminaries. In particular, we state here the combinatorial parameterization of $\operatorname{Stab}\left(h^{\prime}\right)$ invariant tensors (cf. Theorem 3 ) referred to earlier, from which we deduce Theorem 1. In Sect. 4 we prove a sufficient condition for a subalgebra of the tensor algebra to be the algebra of invariants of some reductive subgroup of the orthogonal group, which may be of independent interest. We then use this condition to prove Theorem 3 in Sect. 5. Finally, in Sect. 6 we prove for a special class of vertex models when we can take $h^{\prime}=h$ in the right-hand side of (4). 


\section{Real versus complex}

For real-valued vertex models the following result holds.

Theorem 2 [8] Let $\mathbb{F}=\mathbb{R}$, and let $h \in R^{*}$. Then

$$
\operatorname{rk}\left(M_{f_{h}, k}\right)=\operatorname{dim}\left(V^{\otimes k}\right)^{\operatorname{Stab}(h)} .
$$

The proof of this theorem uses the fact that the orthogonal group of the standard symmetric bilinear form on $\mathbb{R}^{n}$ is compact; the analogous statement for noncompact forms is not true. Over algebraically closed fields of characteristic zero, all nondegenerate forms are equivalent. Consequently, we cannot simply take $h^{\prime}=h$ in Theorem 1 , as the following example shows.

Example Let $i$ be a square root of -1 and set $n:=2, x_{1}:=x, x_{2}:=y$. Consider the vertex model $h: \mathbb{F}[x, y] \rightarrow \mathbb{F}$ given by

$$
h\left(x^{a} y^{b}\right)= \begin{cases}1 & \text { if } a=1 \text { and } b=0 \\ i & \text { if } a=0 \text { and } b=1 \\ 0 & \text { else. }\end{cases}
$$

Note that for any graph $G$ with at least one vertex, we have $f_{h}(G)=0$. Indeed, if $G$ contains an isolated vertex or a vertex of degree at least 2 , then $f_{h}(G)=0$. Else, $G$ is a perfect matching. Since for an edge $e$, we have $f_{h}(e)=h(x)^{2}+h(y)^{2}=0$, in this case $f_{h}(G)$ also equals 0 . So the rank of $M_{f_{h}, 1}$ is equal to zero. It is not difficult to see that $\operatorname{Stab}(h)=\{I\}$, where $I$ is the identity in $O_{2}$. Hence, $\operatorname{rk}\left(M_{f_{h}, 1}\right) \neq \operatorname{dim} V^{\operatorname{Stab}(h)}=$ 2. More generally, the following holds: $\operatorname{rk}\left(M_{f_{h}, k}\right)=\operatorname{dim}\left(V^{\otimes k}\right)^{O_{2}}$. The vertex model $h^{\prime} \equiv 0 \in \mathbb{F}[x, y]^{*}$ does the job.

\section{Framework and preliminaries}

In the remainder of this paper we assume that $\mathbb{F}$ is algebraically closed and has characteristic zero. Let $\mathbb{F} \mathcal{F}_{k}$ denote the vector space consisting of (finite) formal $\mathbb{F}$-linear combinations of fragments. Extend the gluing operation bilinearly to $\mathbb{F} \mathcal{F}_{k} \times \mathbb{F} \mathcal{F}_{k}$. Let

$$
\mathcal{A}:=\bigoplus_{k=0}^{\infty} \mathbb{F} \mathcal{F}_{k}
$$

Make $\mathcal{A}$ into a graded associative $\mathbb{F}$-algebra by defining, for $F \in \mathcal{F}_{k}$ and $H \in \mathcal{F}_{l}$, the product $F H$ to be the disjoint union of $F$ and $H$, where the open end of $H$ labeled $i$ is relabeled to $k+i$.

Fix a vertex model $h \in R^{*}$. Set $\mathcal{I}_{k}:=\left\{\gamma \in \mathbb{F} \mathcal{F}_{k} \mid f_{h}(\gamma * F)=0\right.$ for all $k$-fragments $F$, and let $\mathcal{I}:=\bigoplus_{k=0}^{\infty} \mathcal{I}_{k}$. Observe that

$$
\operatorname{rk}\left(M_{f_{h}, k}\right)=\operatorname{dim}\left(\mathbb{F} \mathcal{F}_{k} / \mathcal{I}_{k}\right)
$$


Let $T(V):=\bigoplus_{i=0}^{\infty} V^{\otimes k}$ be the tensor algebra of $V$ (with product the tensor product). For $\phi:[k] \rightarrow[n]$, define $e_{\phi}:=e_{\phi(1)} \otimes \cdots \otimes e_{\phi}(k)$. The $e_{\phi}$ form a basis for $V^{\otimes k}$. We will now exhibit a natural homomorphism from $\mathcal{A}$ to $T(V)$.

For a $k$-fragment $F$, we denote its edges (including half edges) by $E F$ and its vertices (not including open ends) by $V F$. Moreover, we will identify the half edges of $F$ with the set $[k]$. Let $F \in \mathcal{F}_{k}$, and let $\phi:[k] \rightarrow[n]$. Define

$$
h_{\phi}(F):=\sum_{\substack{\psi: E F \rightarrow[n] \\ \psi(i)=\phi(i)}} \prod_{\substack{i=1, \ldots, k}} h\left(\prod_{e \in V F} x_{\psi(e)}\right) .
$$

We can now define the map $p_{h}: \mathcal{A} \rightarrow T(V)$ by

$$
F \mapsto \sum_{\phi:[k] \rightarrow[n]} h_{\phi}(F) e_{\phi}
$$

for $F \in \mathcal{F}_{k}$, extended linearly to $\mathcal{A}$. Observe that for $F \in \mathcal{F}_{0}, p_{h}(F)=f_{h}(F)$, and note that for $F, H \in \mathcal{F}_{k}$,

$$
f_{h}(F * H)=\sum_{\phi:[k] \rightarrow[n]} h_{\phi}(F) h_{\phi}(H) .
$$

It is not difficult to see that $p_{h}$ is a homomorphism of algebras. By (11) it follows that $\operatorname{ker} p_{h} \subseteq \mathcal{I}$. This gives rise to the following definition: we call $h \in R^{*}$ nondegenerate if $\operatorname{ker} p_{h}=\mathcal{I}$. Equivalently, $h \in R^{*}$ is nondegenerate if the algebra $p_{h}(\mathcal{A})$ is nondegenerate with respect to the bilinear form on $T(V)$ induced by that on $V$. So for nondegenerate $h$, we have $\mathcal{A} / \mathcal{I} \cong p_{h}(\mathcal{A})$ and in particular, by (8),

$$
\operatorname{rk}\left(M_{f_{h}, k}\right)=\operatorname{dim}\left(p_{h}(\mathcal{A}) \cap V^{\otimes k}\right) .
$$

The following theorem gives a combinatorial parameterization of the tensors invariant under $\operatorname{Stab}\left(h^{\prime}\right)$. By (12), it implies Theorem 1.

Theorem 3 Let $h \in R^{*}$. Then there exists a nondegenerate $h^{\prime} \in R^{*}$ with $f_{h}(G)=$ $f_{h^{\prime}}(G)$ for all $G \in \mathcal{G}$ such that

$$
p_{h^{\prime}}(\mathcal{A})=T(V)^{\mathrm{Stab}\left(h^{\prime}\right)} .
$$

We will give a proof of Theorem 3 in Sect. 5. The next section deals with some preparations for the proof.

Remark 2 Following Remark 1, the map $p_{h}$ can be understood in a coordinate-free manner as follows. As before, a $k$-fragment $F$ gives rise to a polynomial function $\psi: V^{[k] \cup V F} \rightarrow \mathbb{F}$. Since open ends have degree one, this map is multilinear in the arguments labeled by $[k]$. Hence, $\psi$ sits naturally in $\left(V^{*}\right)^{\otimes k} \otimes R^{\otimes V F}$. Applying $1 \otimes$ $h^{\otimes V F}$ to it gives an element of $\left(V^{*}\right)^{\otimes k}$, which is also an element of $V^{\otimes k}$ by the natural map $V^{*} \rightarrow V$ induced by the bilinear form. This is the element $p_{h}(F)$. By definition the function $\psi$ is $O_{n}$-invariant. This implies that for all $g \in O_{n}$, we have $g p_{h}(F)=p_{g h}(F)$. 


\section{Algebras of invariant tensors}

The proof in [8] of Theorem 2 depends on a result of Schrijver [10] characterizing which subalgebras of the real tensor algebra arise as invariant algebras of subgroups of the real orthogonal group. Here we prove a variant of Schrijver's result valid over an algebraically closed field $\mathbb{F}$ of characteristic zero.

Below we state and prove a sufficient condition for a subalgebra of $T(V)$ to be the algebra of $H$-invariants for some reductive group $H \subseteq O_{n}$. Derksen (private communication, 2006) completely characterized the subalgebras of $T(V)$ that are the algebras of $H$-invariant tensors for some reductive group $H \subseteq O_{n}$, but we do not need the full strength of his result to prove Theorem 3 .

First we introduce some terminology. For $1 \leq i<j \leq k \in \mathbb{N}$, the contraction $C_{i, j}^{k}$ is the unique linear map

$$
\begin{aligned}
& C_{i, j}^{k}: V^{\otimes k} \rightarrow V^{\otimes k-2} \quad \text { satisfying } \\
& v_{1} \otimes \cdots \otimes v_{k} \mapsto\left\langle v_{i}, v_{j}\right\rangle v_{1} \otimes \cdots \otimes v_{i-1} \otimes v_{i+1} \cdots \otimes v_{j-1} \otimes v_{j+1} \otimes \cdots \otimes v_{k} .
\end{aligned}
$$

Contractions are clearly $O_{n}$-equivariant, and so are compositions of contractions. We will also use $\langle\cdot, \cdot\rangle$ for the induced symmetric bilinear form on tensor powers $V^{\otimes k}$ of $V$. This bilinear form is the composition of the tensor product $V^{\otimes k} \times V^{\otimes k} \rightarrow V^{\otimes 2 k}$ followed by $C_{1, k+1}^{2 k}, C_{1, k}^{2 k-2}$, etc. up to $C_{1,2}^{2}$.

A graded subspace $A$ of $T(V)$ is called contraction closed if $C_{i, j}^{k}(a) \in A$ for all $a \in A \cap V^{\otimes k}$ and $i<j \leq k \in \mathbb{N}$. Note that for any subgroup $H \subseteq O_{n}, T(V)^{H}$ is a graded and contraction closed subalgebra of $T(V)$.

Theorem 4 Let $A \subseteq T(V)$ be a graded contraction closed subalgebra containing $\sum_{i=1}^{n} e_{i} \otimes e_{i}$. If the pointwise stabilizer $\operatorname{Stab}(A):=\bigcap_{a \in A} \operatorname{Stab}(a)$ equals $\operatorname{Stab}(w)$ for some $w \in A$ whose $O_{n}$-orbit is closed in the Zariski topology, then $A=T(V)^{\operatorname{Stab}(A)}$, and moreover $\operatorname{Stab}(A)$ is a reductive group.

Proof Let $w \in A$ be such that $H:=\operatorname{Stab}(w)$ equals $\operatorname{Stab}(A)$. Write $w=w_{1}+\cdots+$ $w_{t}$ with $w_{j} \in W_{j}:=V^{\otimes n_{j}}$ the homogeneous components of $w$, and assume that $O_{n} w \subseteq W:=\bigoplus_{j=1}^{t} W_{j}$ is closed. The map $O_{n} \rightarrow W$ given by $g \mapsto g w$ induces an isomorphism $O_{n} / H \rightarrow O_{n} w$ of quasi-affine varieties (cf. [5, Sect. 12]). As $O_{n} w$ is closed, both varieties are affine, and moreover, regular functions on $O_{n} w$ extend to regular functions (polynomials) on $W$. So they are generated by $W_{j}^{*}$ for $j=1, \ldots, t$. This means that any regular function on $O_{n} / H$ is a linear combination of functions of the form

$$
g H \mapsto\left\langle g w_{1}, u_{1}\right\rangle^{d_{1}} \cdots\left\langle g w_{t}, u_{t}\right\rangle^{d_{t}},
$$

where $d_{1}, \ldots, d_{t}$ are natural numbers, and $u_{j} \in W_{j}$ for all $j$. Note that the right-hand side of (15) can be obtained from

$$
\left(g w_{1}^{\otimes d_{1}}\right) \otimes \cdots \otimes\left(g w_{t}^{\otimes d_{t}}\right) \otimes u_{1}^{\otimes d_{1}} \otimes \cdots \otimes u_{t}^{\otimes d_{t}}
$$

by repeatedly contracting the $g w_{j}$ with the corresponding $u_{j}$. Since $A$ is an algebra, the tensor products of the $w_{j}$ lie in $A$. More succinctly, we find that every regular 
function on $O_{n} / H$ is a linear combination of functions of the form $g \mapsto K[(g q) \otimes$ $u]=K\left[q \otimes\left(g^{-1} u\right)\right]$ with $u \in T(V)$ and $q \in A$ in the same graded piece of $T(V)$ and $K$ a composition of contractions.

Clearly, $A$ is contained in $T(V)^{H}$. To prove the converse, let $a \in\left(V^{\otimes k}\right)^{H}$. Let $z_{1}, \ldots, z_{s}$ be a basis of $V^{\otimes k}$. Then we can write

$$
g a=\sum_{i=1}^{s} f_{i}(g) z_{i}
$$

for all $g \in O_{n}$, where the $f_{i}$ are regular functions on $O_{n}$. Since $g h a=g a$ for all $h \in H$, it follows that the $f_{i}$ induce regular functions on $O_{n} / H$. By the above, for each $i=1, \ldots, s$, we can write

$$
f_{i}(g)=\sum_{j} K_{i, j}\left[q_{i, j} \otimes g^{-1} u_{i, j}\right]
$$

for certain $q_{i, j} \in A$ and $u_{i, j} \in T(V)$ and sequences $K_{i, j}$ of contractions. Multiplying both sides of (17) by $g^{-1}$, we get

$$
a=\sum_{i, j} K_{i, j}\left[q_{i, j} \otimes\left(g^{-1} u_{i, j}\right) \otimes\left(g^{-1} z_{i}\right)\right],
$$

where we have abused the notation $K_{i, j}$ to stand for the same series of contractions as before, but leaving the last $k$ tensor factors $V$ (containing $g^{-1} z_{i}$ ) intact. Let $\rho$ be the Reynolds operator of $O_{n}$. Then we have

$$
a=\sum_{i, j} K_{i, j}\left[q_{i, j} \otimes \rho\left(u_{i, j} \otimes z_{i}\right)\right] .
$$

In the case where $\mathbb{F}=\mathbb{C}$ this follows immediately by integrating (19) over $g$ in the compact real orthogonal group (with respect to the Haar measure). In the general case this follows from standard properties of the Reynolds operator, which we omit.

To complete the proof, note that $q_{i, j} \in A$ and $\rho\left(u_{i, j} \otimes z_{i}\right) \in T(V)^{O_{n}}$. Now by the First Fundamental Theorem for the orthogonal group (see, for example, [11, Sect. 5.3.2]), $T(V)^{O_{n}}$ is the smallest contraction-closed graded subalgebra of $T(V)$ containing $\sum_{i} e_{i} \otimes e_{i}$ and hence is contained in $A$. As $A$ is a graded and contraction closed subalgebra of $T(V)$, it follows that $a \in A$. Finally, since $O_{n} / H$ is affine, Matsushima's criterion (see [1] for an elementary proof) implies that $\operatorname{Stab}(A)=H$ is reductive.

\section{A proof of Theorem 3}

In this section we give a proof of Theorem 3. First we show some results from [8] allowing us to apply Theorem 4 . For completeness, we will include the proofs.

We define a contraction operation for fragments. For $1 \leq i<j \leq k \in \mathbb{N}$, the contraction $\Gamma_{i, j}^{k}: \mathcal{F}_{k} \rightarrow \mathcal{F}_{k-2}$ is defined as follows: for $F \in \overline{\mathcal{F}}_{k}, \Gamma_{i, j}^{k}(\bar{F})$ is the $(k-2)$ fragment obtained from $F$ by connecting the half edges incident with the open ends 
labeled $i$ and $j$ into one single edge (without labeled vertex) and then relabeling the remaining open ends $1, \ldots, k-2$ so that the order is preserved.

The following lemma shows that $p_{h}$ preserves contractions.

Lemma 1 For $1 \leq i<j \leq k \in \mathbb{N}$ and $F \in \mathcal{F}_{k}$,

$$
p_{h}\left(\Gamma_{i, j}^{k}(F)\right)=C_{i, j}^{k}\left(p_{h}(F)\right) .
$$

Proof Let $1 \leq i<j \leq k$, and let $F \in \mathcal{F}_{k}$. Note that for $\phi:[k] \rightarrow[n]$, the contraction of $e_{\phi}$ is contained in $\left\{e_{\psi} \mid \psi:[k-2] \rightarrow[n]\right\}$ if $\phi(i)=\phi(j)$ and is zero otherwise. The following equalities now prove the lemma:

$$
\begin{aligned}
C_{i, j}^{k}\left(p_{h}(F)\right) & =\sum_{\phi:[k] \rightarrow[n]} h_{\phi}(F) C_{i, j}^{k}\left(e_{\phi}\right)=\sum_{\substack{\phi:[k] \rightarrow[n] \\
\phi(i)=\phi(j)}} h_{\phi}(F) C_{i, j}^{k}\left(e_{\phi}\right) \\
& =\sum_{\psi:[k-2] \rightarrow[n]} h_{\psi}\left(\Gamma_{i, j}^{k}(F)\right) e_{\psi}=p_{h}\left(\Gamma_{i, j}^{k}(F)\right) .
\end{aligned}
$$

The next proposition shows that $\operatorname{Stab}(h)$ is equal to the pointwise stabilizer of $p_{h}(\mathcal{A})$.

Proposition 1 Let $h \in R^{*}$. Then $\operatorname{Stab}(h)=\operatorname{Stab}\left(p_{h}(\mathcal{A})\right)$.

Before we give a proof, we introduce some terminology. The basic $k$-fragment $F_{k}$ is the $k$-fragment that contains one vertex and $k$ open ends connected to this vertex, labeled 1 up to $k$. For a map $\phi:[k] \rightarrow[n]$, we define the monomial $x^{\phi} \in R$ by $x^{\phi}:=\prod_{i=1}^{k} x_{\phi(i)}$. It is not difficult to see that

$$
h_{\phi}\left(F_{k}\right)=h\left(x^{\phi}\right)
$$

Proof of Proposition 1 By Remark 2 we have for all $F \in \mathcal{F}_{k}$ and $g \in O_{n}$ that

$$
g p_{h}(F)=p_{g h}(F) .
$$

This immediately implies that $\operatorname{Stab}(h) \subseteq \operatorname{Stab}\left(p_{h}(\mathcal{A})\right)$. To see the opposite inclusion, consider (24) for basic fragments. Using (23), we find that $g \in \operatorname{Stab}\left(p_{h}(\mathcal{A})\right)$ implies that $g h=h$.

Now we can give a proof of Theorem 3 .

Proof of Theorem 3 The first step of the proof uses some ideas of [3]. Consider

$$
S:=\mathbb{F}\left[y_{\alpha} \mid \alpha \in \mathbb{N}^{n}\right]
$$

a polynomial ring in the infinitely many variables $y_{\alpha}$. These variables are in bijective correspondence with the monomials of $R$ via $y_{\alpha} \leftrightarrow x_{1}^{\alpha_{1}} \cdots x_{n}^{\alpha_{n}}$. Let $\mathbb{N}_{d}^{n}=\left\{\alpha \in \mathbb{N}^{n} \mid\right.$ 
$|\alpha| \leq d\}$, and let $S_{d} \subset S$ be the ring of polynomials in the variables $y_{\alpha}$ with $\alpha \in \mathbb{N}_{d}^{n}$. Furthermore, let $\mathcal{G}_{d}$ be the set of all graphs of maximum degree $d$. Let $\pi: \mathbb{F} \mathcal{G} \rightarrow S$ be the linear map defined by

$$
G \mapsto \sum_{\phi: E G \rightarrow[n]} \prod_{v \in V G} y_{\phi}(\delta(v))
$$

for any $G \in \mathcal{G}$, where we consider the multiset $\phi(\delta(v))$ as an element of $\mathbb{N}^{n}$. Note that $\pi(G)(y)=f_{y}(G)$ for all $G \in \mathcal{G}$.

The orthogonal group acts on $S$ via the bijection between the variables of $S$ and the monomials of $R$. Then, as was observed by Szegedy [12] (see also [3]), for any $d$,

$$
\pi\left(\mathbb{F} \mathcal{G}_{d}\right)=S_{d}^{O_{n}} .
$$

Let

$$
\begin{aligned}
Y_{d} & :=\left\{y \in \mathbb{F}^{\mathbb{N}_{d}^{n}} \mid \pi(G)(y)=f_{h}(G) \text { for all } G \in \mathcal{G}_{d}\right\}, \\
Y & :=\left\{y \in \mathbb{F}^{\mathbb{N}^{n}} \mid \pi(G)(y)=f_{h}(G) \text { for all } G \in \mathcal{G}\right\} .
\end{aligned}
$$

Then $Y$ is nonempty, as it contains $h$. Moreover, by (27) the variety $Y_{d}$ is a fiber of the quotient map $\mathbb{F}^{\mathbb{N}_{d}^{n}} \rightarrow \mathbb{F}^{\mathbb{N}_{d}^{n}} / / O_{n}$. In particular, $Y_{d}$ contains a unique closed orbit $C_{d}$ (cf. [5, Sect. 8.3] or [6, Satz 3, p. 101]).

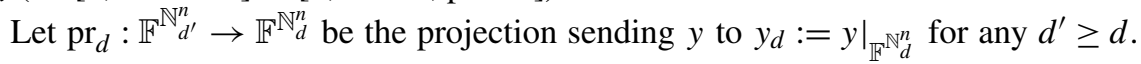
Note that $\operatorname{pr}_{d}\left(Y_{d^{\prime}}\right) \subseteq Y_{d}$ for $d^{\prime} \geq d$. Since $\operatorname{pr}_{d}\left(C_{d^{\prime}}\right)$ is an $O_{n}$-orbit, its closure contains $C_{d}$. Hence, we have

$$
\operatorname{dim} C_{d} \leq \operatorname{dim} \operatorname{pr}_{d}\left(C_{d^{\prime}}\right) \leq \operatorname{dim} C_{d^{\prime}},
$$

where the left-most inequality is strict unless $\operatorname{pr}_{d}\left(C_{d^{\prime}}\right)$ equals the closed orbit $C_{d}$. As $\operatorname{dim} C_{d}$ is bounded from above by $\operatorname{dim} O_{n}$ for all $d$, we may choose $d_{0}$ where the former dimension reaches its maximal value. Then for $d^{\prime} \geq d \geq d_{0}$, both inequalities in (29) are equalities, and we have $\operatorname{pr}_{d}\left(C_{d^{\prime}}\right)=C_{d}$. We therefore find a sequence $h_{d_{0}}^{\prime} \in$ $C_{d_{0}}, h_{d_{0}+1}^{\prime} \in C_{d_{0}+1}, \ldots$ in which every next element is projected onto the previous one. That the element $h^{\prime} \in \mathbb{F}^{\mathbb{N}^{n}}$ determined by $\operatorname{pr}_{d}\left(h^{\prime}\right)=h_{d}^{\prime} \in C_{d}$ for all $d \geq d_{0}$ has the property that $f_{h^{\prime}}=f_{h}$ follows from the fact that each $h_{d}^{\prime}$ lies in $Y_{d}$.

We will now show that this $h^{\prime}$ is as required. First note that $\operatorname{Stab}\left(h^{\prime}\right)=$ $\cap_{e \geq 0} \operatorname{Stab}\left(h_{e}^{\prime}\right)$. Since the ring of regular functions of $O_{n}$ is Noetherian, it follows that there exists $e$ such that $\operatorname{Stab}\left(h^{\prime}\right)=\operatorname{Stab}\left(h_{e}^{\prime}\right)$. We may assume that $e \geq d_{0}$. Let $F=\sum_{0 \leq k \leq e} F_{k}$, the sum in $\mathcal{A}$ of the first $e+1$ basic fragments. Then

$$
\operatorname{Stab}\left(p_{h^{\prime}}(F)\right)=\operatorname{Stab}\left(h^{\prime}\right) .
$$

Write $w=p_{h^{\prime}}(F)$, and note that $w$ is the image of $\operatorname{pr}_{e}\left(h^{\prime}\right)$ under the natural embedding of $\mathbb{F}^{\mathbb{N}_{e}^{n}}$ into $\bigoplus_{k=0}^{e} V^{\otimes k}$. In particular, we can view $C_{e}$ and $Y_{e}$ as subvarieties of $\bigoplus_{k=0}^{e} V^{\otimes k}$.

It is clear that $p_{h^{\prime}}(\mathcal{A})$ is a graded algebra. It is contraction closed by Lemma 1 . By Proposition 1 we have that $\operatorname{Stab}\left(p_{h^{\prime}}(\mathcal{A})\right)=\operatorname{Stab}(w)$. Moreover, the orbit of $w$ is Zariski closed, as the orbit of $\operatorname{pr}_{e}\left(h^{\prime}\right)$ is the unique closed orbit $C_{e}$ in $Y_{e}$. Also, we have $\sum_{i} e_{i} \otimes e_{i} \in p_{h^{\prime}}(\mathcal{A})$ as it is the image of the edge whose both endpoints are open ends. So we can apply Theorem 4 to find that $p_{h^{\prime}}(\mathcal{A})=T(V)^{\operatorname{Stab}\left(h^{\prime}\right)}$. More- 
over, we find that $\operatorname{Stab}\left(h^{\prime}\right)$ is reductive. From this we conclude that $h^{\prime}$ is nondegenerate. Indeed, suppose that $p_{h^{\prime}}(\gamma) \neq 0$ for some $\gamma \in \mathcal{A}$. Then there exists $v \in T(V)$ such that $\left\langle p_{h^{\prime}}(\gamma), v\right\rangle \neq 0$. Since $\operatorname{Stab}\left(h^{\prime}\right)$ is reductive, we can write $v=v_{1}+v_{2}$ with $v_{1} \in T(V)^{\mathrm{Stab}\left(h^{\prime}\right)}$ and $v_{2}$ in a different isotypic component. Using Schur's lemma and the fact that $O_{n}$ preserves the bilinear form, we find that $\left\langle p_{h^{\prime}}(\gamma), v_{2}\right\rangle=0$. As $v_{1} \in p_{h^{\prime}}(\mathcal{A})$, it follows that $h^{\prime}$ is nondegenerate.

Remark 3 The elements $h^{\prime} \in R^{*}$ with the property that for sufficiently large $d$, the projection $h_{d}^{\prime}$ lies in the unique closed orbit in the closure of the orbit of $h_{d}$ form a single orbit under the orthogonal group. This follows from the slightly stronger observation that for $d$ sufficiently large and $d^{\prime} \geq d$, the projection from $C_{d^{\prime}}$ to $C_{d}$ is not only surjective and dimension preserving, but even an isomorphism (since by Noetherianity point stabilizers cannot shrink indefinitely).

\section{One-parameter groups and spin models}

There is a beautiful criterion for closeness of orbits involving one-parameter subgroups of $O_{n}$, i.e., homomorphisms $\lambda: \mathbb{F}^{*} \rightarrow O_{n}$ of algebraic groups. For any such homomorphism, there exists a basis $v_{1}, \ldots, v_{n}$ of $V$ such that $\left\langle v_{i}, v_{j}\right\rangle=\delta_{n+1-i, j}$ (so that the Gram matrix of the basis has zeroes everywhere except ones on the longest anti-diagonal; we will call such bases canonical) and such that $\lambda(t) v_{i}=t^{d_{i}} v_{i}$ for some integral weights $d_{1} \geq \cdots \geq d_{n}$ satisfying $d_{i}=-d_{n+1-i}$ for all $i$. This follows, for instance, from [2, §23.4] (ignoring the subtle rationality issues there as $\mathbb{F}$ is algebraically closed) and the fact that all maximal tori are conjugate [2, $\S 11.3]$. Conversely, given a canonical basis $v_{1}, \ldots, v_{n}$ and such a sequence of $d_{i} \mathrm{~s}$, the $\lambda: \mathbb{F}^{*} \rightarrow O_{n}$ defined by $\lambda(t) v_{i}=t^{d_{i}} v_{i}$ is a one-parameter subgroup of $O_{n}$.

The criterion alluded to is that whenever $W$ is a finite-dimensional $O_{n}$-module and $w$ is an element of $W$, there exists a one-parameter subgroup $\lambda$ such that $\lim _{t \rightarrow 0} \lambda(t) w$ exists and lies in the unique closed orbit in the closure of $O_{n} w$ (for example, see [7, Theorem 6.9]). Here the existence of the limit by definition means that the morphism $\mathbb{F}^{*} \rightarrow W, t \mapsto \lambda(t) w$ extends to $\mathbb{F}$. It then does so in a unique manner, and the value at 0 is declared the limit. Put differently, just like $V$, the module $W$ decomposes into a direct sum of weight spaces, and the condition is that all components of $w$ in $\lambda$-weight spaces corresponding to negative weights are zero, and the component of $w$ in the zero weight space is the limit.

Example In our example on p. 396, $h \in R^{*}=\left(\bigoplus_{e} S^{e} V^{*}\right)^{*}$ is zero on all graded pieces $S^{e} V^{*}$ except on $S^{1} V^{*}=V^{*}$. The restriction of $h$ to that space is an element of $\left(V^{*}\right)^{*}=V$, namely, equal to $v_{1}:=e_{1}+i e_{2}$. This is an isotropic vector relative to the bilinear form, and so is its complex conjugate $v_{2}:=e_{1}-i e_{2}$. The linear map $V \rightarrow V$ scaling $v_{1}$ with $t \in \mathbb{F}$ and $v_{2}$ with $t^{-1}$ is an element of the orthogonal group. Explicitly, this gives the one-parameter subgroup

$$
\lambda: t \mapsto \frac{1}{2 t}\left[\begin{array}{cc}
1+t^{2} & i-i t^{2} \\
-i+i t^{2} & 1+t^{2}
\end{array}\right] \in O_{2}
$$

with the property that $\lim _{t \rightarrow 0} \lambda(t) h_{e}=0$ for all 0 . 
We will now apply the one-parameter group criterion to an important class of vertex models whose partition functions include the partition functions of so-called spin models (cf. [4]) as was shown by Szegedy [12]. Let $u_{1}, \ldots, u_{m}$ be distinct vectors in $V$, and let $a_{1}, \ldots, a_{m}$ be nonzero elements of $\mathbb{F}$. Then define

$$
h(p):=\sum_{i=1}^{m} a_{i} p\left(u_{i}\right)
$$

for $p \in R$. This $h \in R^{*}$ is a vertex model, and we write $h_{e}$ for the restriction of $h$ to polynomials of degree at most $e$. We have the following characterization.

Theorem 5 The orbit $O_{n} h_{e}$ is closed for sufficiently large e if and only if the $O_{n}$ orbit of $\left(u_{1}, \ldots, u_{m}\right)$ in $V^{m}$ is closed, and this happens if and only if the restriction of the bilinear form to the span of the $u_{i}$ is nondegenerate.

Proof The second equivalence is well known, but we include an argument as a warmup for the first equivalence. First, let $U \subseteq V$ be the span of the $u_{i}$. If the restriction of the form to $U$ is degenerate, then we may choose a canonical basis $v_{1}, \ldots, v_{n}$ of $V$ such that $U$ is spanned by $v_{a}, \ldots, v_{b}$ with $b<n+1-a$ (in particular, $v_{a}$ then lies in the radical of the restriction of the form to $U$ ). Now let $\lambda: \mathbb{F}^{*} \rightarrow O_{n}$ be the one-parameter group with $\lambda(t) v_{j}=t v_{j}$ for $j \leq a, \lambda(t) v_{j}=v_{j}$ for $a<j<n+1-a$, and $\lambda(t) v_{j}=t^{-1} v_{j}$ for $j \geq n+1-a$. Then $\lim _{t \rightarrow 0} \lambda(t) u$ exists for all $u \in U$ and lies in the span of $v_{a+1}, \ldots, v_{b}$, a proper subspace of $U$. Hence, $\lim _{t \rightarrow 0} \lambda(t)\left(u_{1}, \ldots, u_{m}\right)$ does not lie in the orbit of $\left(u_{1}, \ldots, u_{m}\right)$, and the latter orbit is not closed.

For the converse, assume that the restriction of the form to $U$ is nondegenerate. By the one-parameter group criterion, to prove the closeness of $O_{n}\left(u_{1}, \ldots, u_{m}\right)$, it suffices to prove that whenever $\lambda$ is a one-parameter subgroup of $O_{n}$ for which the limit $\lim _{t \rightarrow 0} \lambda(t)\left(u_{1}, \ldots, u_{m}\right)=\left(u_{1}^{\prime}, \ldots, u_{m}^{\prime}\right)$ exists, that limit actually lies in the orbit of $\left(u_{1}, \ldots, u_{m}\right)$. Now since the Gram matrix of $\left(u_{1}^{\prime}, \ldots, u_{m}^{\prime}\right)$ equals that of $\left(u_{1}, \ldots, u_{m}\right)$, and since its rank equals the dimension of $U$, we find that the span $U^{\prime}$ of the $u_{i}^{\prime}$ is again a nondegenerate subspace of $V$ of the same dimension as $U$. Hence, the stabilizers of $\left(u_{1}, \ldots, u_{m}\right)$ and $\left(u_{1}^{\prime}, \ldots, u_{m}^{\prime}\right)$ are the isomorphic groups $O\left(U^{\perp}\right)$ and $O\left(\left(U^{\prime}\right)^{\perp}\right)$. In particular, they have the same dimension. Since orbits at the "boundary" of an orbit have strictly larger-dimensional point stabilizers, we conclude that $\left(u_{1}^{\prime}, \ldots, u_{m}^{\prime}\right)$ is in the $O_{n}$-orbit of $\left(u_{1}, \ldots, u_{m}\right)$.

Now for the first part of the theorem, one direction is easy: if the orbit of $\left(u_{1}, \ldots, u_{m}\right)$ is not closed, then there exists a one-parameter subgroup $\lambda: \mathbb{F}^{*} \rightarrow O_{n}$ such that $\lambda(t) u_{i} \rightarrow u_{i}^{\prime} \in V, i=1, \ldots, m$, as $t \rightarrow 0$ and such that $\left(u_{1}^{\prime}, \ldots, u_{m}^{\prime}\right)$ does not lie in the orbit of $\left(u_{1}, \ldots, u_{m}\right)$. Then $\lim _{t \rightarrow 0} h_{e}=: h_{e}^{\prime}$ exists and maps any polynomial $p$ of degree at most $e$ to $\sum_{i=1}^{m} a_{i} p\left(u_{i}^{\prime}\right)$. It is not hard to see that for sufficiently large $e$, the restriction $h_{e}^{\prime}$ is not in the orbit of $h_{e}$, roughly because the set of points $u_{1}, \ldots, u_{m}$ can be recovered from $h$ in an $O_{n}$-equivariant manner (as the set of points defined by the largest ideal of $R$ contained in the kernel of $h$ ), and then so can the $a_{i}$ by plugging in Lagrange interpolation polynomials at the $u_{i}$.

For the converse, assume that $u_{1}, \ldots, u_{m}$ span a nondegenerate subspace $U$ of $V$. We will prove that the orbit of $h_{e}$ is closed for $e \geq 3 m$. Let $\lambda: \mathbb{F}^{*} \rightarrow O_{n}$ be a oneparameter subgroup such that $\lim _{t \rightarrow 0} \lambda(t) h_{e}$ exists; we will show that it lies in the 
orbit of $h_{e}$. Let $v_{1}, \ldots, v_{n}$ be a canonical basis of $V$ with $\lambda(t) v_{j}=t^{d_{j}} v_{j}$ for weights $d_{1} \geq \cdots \geq d_{n}$. Let $x_{1}, \ldots, x_{n}$ be the basis of $V^{*}$ dual to $v_{1}, \ldots, v_{n}$. For any monomial $x^{\alpha}, \alpha \in \mathbb{N}^{n}$, we have

$$
(\lambda(t) h)\left(x^{\alpha}\right)=h\left(\lambda(t)^{-1} x^{\alpha}\right)=h\left(t^{\alpha_{1} d_{1}+\cdots+\alpha_{n} d_{n}} x^{\alpha}\right)=t^{\alpha \cdot d} \sum_{i=1}^{m} a_{i} x^{\alpha}\left(u_{i}\right) .
$$

By assumption, if $x^{\alpha}$ is a monomial of degree at most $e$, the limit as $t \rightarrow 0$ of this expression exists. If $\alpha \cdot d=\alpha_{1} d_{1}+\cdots+\alpha_{n} d_{n}$ is negative, this means that $\sum_{i=1}^{m} a_{i} x^{\alpha}\left(u_{i}\right)$ must be zero. By taking linear combinations, this implies that for any polynomial $p$ of degree at most $e$ in which only monomials $x^{\alpha}$ with $\alpha \cdot d<0$ appear, we have $h(p)=\sum_{i=1}^{m} a_{i} p\left(u_{i}\right)=0$.

In what follows, we exclude the trivial cases where $m=0$ and where $m=1$ and $u_{1}$ is the zero vector; in both of these cases, the orbit of $h$ is just a single point. Next let $b \in\{1, \ldots, n\}$ be the maximal index with $x_{b}(U) \neq\{0\}$ and order the $u_{i}$ such that $x_{b}\left(u_{1}\right), \ldots, x_{b}\left(u_{l}\right) \neq 0(l>0)$ and $x_{b}\left(u_{l+1}\right), \ldots, x_{b}\left(u_{m}\right)=0$. If $d_{b}$ is nonnegative, then all $u_{i}$ lie in the sum of the weight spaces with nonnegative weights, so that $\lim _{t \rightarrow 0} \lambda(t)\left(u_{1}, \ldots, u_{m}\right)$ exists, and by the second equivalence we know that it lies in the orbit of $\left(u_{1}, \ldots, u_{m}\right)$. Then also $h_{e}$ and $\lim _{t \rightarrow 0} \lambda(t) h_{e}$ lie in the same orbit. Hence, we may assume that $d_{b}$ is negative (in particular, $b$ is larger than $\frac{n}{2}$ ).

By the maximality of $b$, the coordinates $x_{b+1}, \ldots, x_{n}$ vanish identically on $U$, and this means that $U$ lies in the subspace of $V$ perpendicular to $v_{1}, \ldots, v_{n-b}$. Since $U$ is nondegenerate, it does not contain a nonzero linear combination of $v_{1}, \ldots, v_{n-b}$. This means, in particular, that the coordinates $x_{n-b+1}, \ldots, x_{b}$ together separate the points $u_{1}, \ldots, u_{l}$. Then so do the monomials $x_{b}, x_{n-b+1} x_{b}^{2}, \ldots, x_{b-1} x_{b}^{2}$. Note that the dot product $\alpha \cdot d$ is negative for each of these (e.g., for the second, it equals $d_{n-b+1}+2 d_{b}=d_{b}<0$, and from there the dot product decreases weakly to the right). It follows that there exists a linear combination $p$ of those (at most) cubic monomials for which $p\left(u_{1}\right), \ldots, p\left(u_{l}\right)$ are distinct and nonzero. Then, by the above, the vector $\left(a_{1}, \ldots, a_{l}\right)^{T}$ is in the kernel of the Vandermonde matrix

$$
\left[\begin{array}{ccc}
p\left(u_{1}\right) & \cdots & p\left(u_{l}\right) \\
p\left(u_{1}\right)^{2} & \cdots & p\left(u_{l}\right)^{2} \\
\vdots & & \vdots \\
p\left(u_{1}\right)^{l} & \cdots & p\left(u_{l}\right)^{l}
\end{array}\right],
$$

since the degree of $p^{l}$ is $3 l \leq e$. Hence, $a_{1}, \ldots, a_{l}$ are all zero, contrary to the assumption that all $a_{i}$ are nonzero. This proves that the orbit of $h_{e}$ is closed for $e \geq 3 \mathrm{~m}$.

An immediate consequence of this result and of the proof of Theorem 1 is the following; we omit the proof of the implication.

Corollary 1 If $u_{1}, \ldots, u_{m}$ span $V, a_{1}, \ldots, a_{m}$ are nonzero elements of $\mathbb{F}$, and $h$ is defined as above, then for all $k$, the rank of $M_{f_{h}, k}$ equals $\operatorname{dim}\left(V^{\otimes k}\right)^{H}$, where $H$ is the finite group consisting of all orthogonal transformations of $V$ mapping each $u_{i}$ to some $u_{j}$ with $a_{j}$ equal to $a_{i}$. 
Acknowledgements The second author thanks Lex Schrijver for useful discussions on an alternative proof for Theorem 3. The first author is supported by a Vidi grant from the Netherlands Organisation for Scientific Research (NWO).

\section{References}

1. Arzhantsev, I.V.: Invariant ideals and Matsushima's criterion. Commun. Algebra 36(12), 4368-4374 (2008)

2. Borel, A.: Linear Algebraic Groups. Springer, New York (1991)

3. Draisma, J., Gijswijt, D.C., Lovász, L., Regts, G., Schrijver, A.: Characterizing partition functions of the vertex model. J. Algebra 350, 197-206 (2012)

4. de la Harpe, P., Jones, V.F.R.: Graph invariants related to statistical mechanical models: examples and problems. J. Comb. Theory, Ser. B 57, 207-227 (1993)

5. Humphreys, J.E.: Linear Algebraic Groups. Graduate Texts in Mathematics, vol. 21. Springer, New York (1975)

6. Kraft, H.: Geometrische Methoden in der Invariantentheorie. Vieweg, Braunschweig (1984)

7. Popov, V.L., Vinberg, E.B.: Invariant Theory, Part II of Algebraic Geometry IV: Linear Algebraic Groups, Invariant Theory. Encyclopedia of Mathematical Sciences, vol. 55. Springer, Berlin (1994)

8. Regts, G.: The rank of edge connection matrices and the dimension of algebras of invariant tensors. Eur. J. Comb. 33, 1167-1173 (2012)

9. Schrijver, A.: Characterizing partition functions of the vertex model by rank growth. http:// homepages.cwi.nl/ lex/files/gremrk.pdf

10. Schrijver, A.: Tensor subalgebras and first fundamental theorems in invariant theory. J. Algebra 319, 1305-1319 (2008)

11. Goodman, R., Wallach, N.R.: Symmetry, Representations and Invariants. Graduate Texts in Mathematics, vol. 255. Springer, Dordrecht (2009)

12. Szegedy, B.: Edge coloring models and reflection positivity. J. Am. Math. Soc. 20(4) (2007) 